than controls, and plasma lactate levels of glucose intolerant FA patients were higher than controls. By intravenous glucose load, glucose responses did not differ significantly between patients and controls. The results showed that FA is associated with insulin resistance, B-cell deficiency, and type I diabetes. The alterations might be genetically linked or metabolically related to the primary defect in FA. (Finocchiaro G et al. Glucose metabolism alterations in Friedreich's ataxia. Neurology August 1988; 38: 1292-96).

COMMENT. Friedreich's heredodegenerative ataxia, incidence of diabetes in intolerance previously, previously

ataxia, the is recessively inherited.

common during oral glucose tests have been reported but the abnormalities of glucose metabolism have not been studied in detail. The abnormalities of glycemic control in FA may be due to the interplay of insulin resistance and $\mathrm{B}-\mathrm{cell}$ dysfunction or their independent effects.

\title{
INFANTILE GANGLIOSIDOSIS
}

Three sisters with infantile-onset 3 GM1 gangliosidosis are reported from the University of Siens, Italy, and the University of Louvain, Brussels, Belgium. The diagnosis was based on the clinical findings of progressive intellectual deterioration by age 6-8 years, ataxia, spastic tetraparesis, and athetoid-choreiform movements; lysosomal vacuoles in CSF, bone marrow, and conjunctiva; and on decreased activity of serum, leukocyte, and fibroblast B-D-galactosidase and abnormal urinary excretion of oligosaccharides. (Guazzi GC et al. Type 3 (chronic) GM1 gangliosidosis presenting as infanto-choreo-athetotic dementia, without epilepsy, in three sisters. Neurology July 1988; $38: 1124-27$ ).

COMmeNT. Gangliosidosis occurs in 3 forms: 1) infantile, characterized by Hurler's facial features, bony abnormalities, hepatoslenomegaly, cherry-red spot, and progressive neurological signs; 2) late infantile-juvenile, without skeletal changes or marked visceromegaly, but severe intellectual deterioration, ataxia, myoclonic seizures, and retinal degeneration; and 3) dystonic juvenile form. The clinical findings in the present report resembled those in the dystonic form except that the intellectual deterioration was more severe.

\section{TOXIC DISORDERS}

\section{FETAL ALCOHOL SYNDROME}

Hearing and speech and language development in 14 children with fetal alcohol syndrome were evaluated at the Fetal Alcohol Research Center, Wayne State University School of Medicine, Detroit, Michigan, and the Audiology Division, School of Medicine, University of Colorado Health Sciences Center, Denver, Colorado. Recurrent serous otitis media with hearing loss and speech and language problems occurred in 13, and 4 also had sensorineural hearing loss. The IQ was 70-85 in 7 children and below 69 in 7 . The authors conclude that 